\title{
Morphological analysis: to evaluate the pattern of residential building based on wind performance
}

\author{
Yihui Yang, Wei You, Yunlong Peng, Wowo Ding* \\ School of Architecture and Urban Planning, Nanjing University, Nanjing, China \\ *(corresponding author) \\ E-mail: yi_hui_yang@outlook.com, youwei@nju.edu.cn,249626020@qq.com,dww@nju.edu.cn
}

\begin{abstract}
Residential morphological patterns are reflection of people's living habits and tradition, local climate and building regulations, so that one of those factors could be studied through in order to understand residential morphological patterns. Based upon our previous study, we do know that in China living habits and local climate mainly influence the shape of residential buildings and apartment patterns, but we do not know whether the pattern of residential plots determined by FAR and sunshine hours are suitable for wind environment related to residential environmental quality. Therefore, it is very significant to evaluate wind environment within residential plots based on the apartment pattern controlled by various building codes. Our study focuses on the pattern of Slab apartments in Nanjing, which are mainly used in China, and selects 40 residential slices with different plot shapes, plot FAR, building heights and sizes. Based upon MATLAB, we have got all geometrical data between buildings among these slices to identify the spatial pattern character of each residential plot. Through evaluating wind environment of these slices by simulation we can obtain wind speed, pressure and age of air and choose the pattern of age of air as the main evaluation factor of wind performance. Correlation analysis will be made between the apartment patterns and pattern of age of air, by doing so, each typical space between buildings will be evaluated. Our study will reveal the relevance of apartment pattern and wind environment, which can be used to support and improve design in the future.
\end{abstract}

Keywords: Residential plot, Apartment pattern, performance evaluation, wind environment

\section{Introduction}

Residential buildings are the largest region in the city, and residential area morphology is an important part of urban morphology which would be affected by living habits, economic levels, cultural traditions and other factors.

Different patterns of residential areas are the most obvious manifestation of the differentiation of residential area morphology. In order to understand and study different patterns, many scholars have studied these from different points of view. This paper will evaluate and analyze the pattern based on performance orientation.
In Chinese residential area, floor area ratio and sunshine distance are two main factors that influence and restrict patterns of residential areas. Because of Chinese traditional culture, people attach great importance to sunlight right. Therefore, the residential buildings in different years have satisfied the sunshine distance of the corresponding period, people can get ideal natural lighting conditions. With the development of modern society, People put higher demands on the quality of living -healthy indoor and outdoor environment. The natural factor that has the greatest effect on health is wind, except sunshine. In today's society, with population density increasing 
and rapid development of city renewal, a large number of high-rise buildings, vehicles and factories cause serious urban heat island effect, higher average temperature, and a large amount of car exhaust, PM2.5 and other pollutants are gathered in cities and cannot be dispersed. A good outdoor wind environment can greatly reduce these negative impact. Thus the indoor and outdoor wind environment has more and more influence on people's health.

Patterns of buildings will have a decisive effect on wind environment, predecessors have done a lot of research, starting from form elements (including buildings' length, width, height, spacing, density, floor area rate and so on) which decide patterns, and proved that form different factors have different effects on the wind environment.

Mfula, et, al. (2005) discussed the influence of building spacing on pollutant dispersion through wind tunnel experiments. Buccolieri et al. (2010) evaluated air exchange rate of different spacing by numeric model. Feng Yang (2013) discussed the relationship between floor area rate, coverage rate and sky view factor these indexes and wind speed. Bady (2007) discussed effects of building height, street width, and patterns on PFR, VF, and TP. Tingting $\mathrm{Hu}$ (2013) discussed relationships between different coverage rate, building height, building pattern and wind speed, VF, pollutant concentrations. Matteo Carpentieri (2015) studied influence of building height on wind speed under specific patterns.

Therefore, this article will evaluate the wind environment of residential area in Nanjing, china, and explore what kind of apartment pattern can produce a better wind environment.

\section{Method}

\section{Morphological object of research}

Oke et al. (1988) proposed different ratio of building height to building spacing $(\mathrm{H} / \mathrm{W})$ that would produce three kinds of typical flow types. According to results of wind tunnel tests, he summed up the function curve of this ratio with different flow types. Oke's classic conclusion that the ratio of building height to building spacing $(\mathrm{H} / \mathrm{W})$ will affect wind environment is confirmed, cited and expanded to varying degrees in following articles. Chan, et, al. (2001) studied effects of different $\mathrm{H} / \mathrm{W}$ and building heights on pollutants at different sites. Simoëns and Wallace (2008) studied diffusion of pollutants at different $\mathrm{H} / \mathrm{W}$. Buccolieri, et, al. (2015) studied effects of different lateral and Longitudinal spacing on wind environment. You and Ding (2016) studied influence of different sizes, spacing, and orientation on ventilation efficiency in residential buildings by numeric model.

Therefore, this paper will also cite this classical theory, using ratio of building height to building spacing, to carry out experiments.

Criteria for determining the healthy wind environment

In the environment field, two important indicators to judge wind environment are pollutant concentration and mean wind speed. However, these indicators have obvious shortcomings. The index of pollutant concentration is complex to calculate and calculated by artificially throwing a c (such as carbon dioxide) in the study area, The initial range of pollutant has a strong effect on the accuracy of results.

The wind speed index is statistical data of wind speed in one area, this index cannot determine the direction of wind, and a region with large wind speed does not necessarily bring about fresh air. Therefore, the wind environment will not necessarily be improved, there may be secondary pollution.

According to needs of architecture, this paper makes a reasonable deduction to simplify the judgment standard of healthy wind environment and assist people in architecture in computing and understanding.

A region with healthy wind conditions is an area of little pollutant concentration, winds should travel across this area quickly to take away the pollutants.

So, the value of wind speed will affect the concentration of pollutants and the direction of wind speed should be in accordance with the simulated wind direction to guarantee fresh wind which can take away the pollutant without 

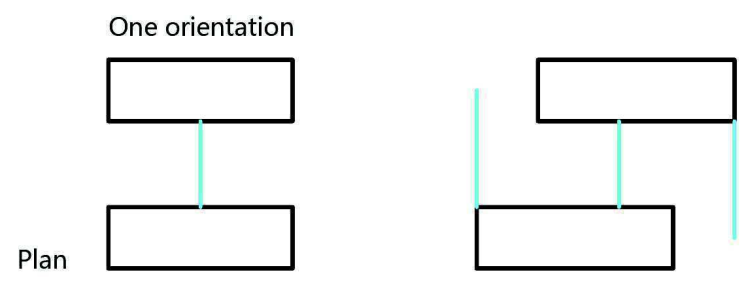

Multi-orientation
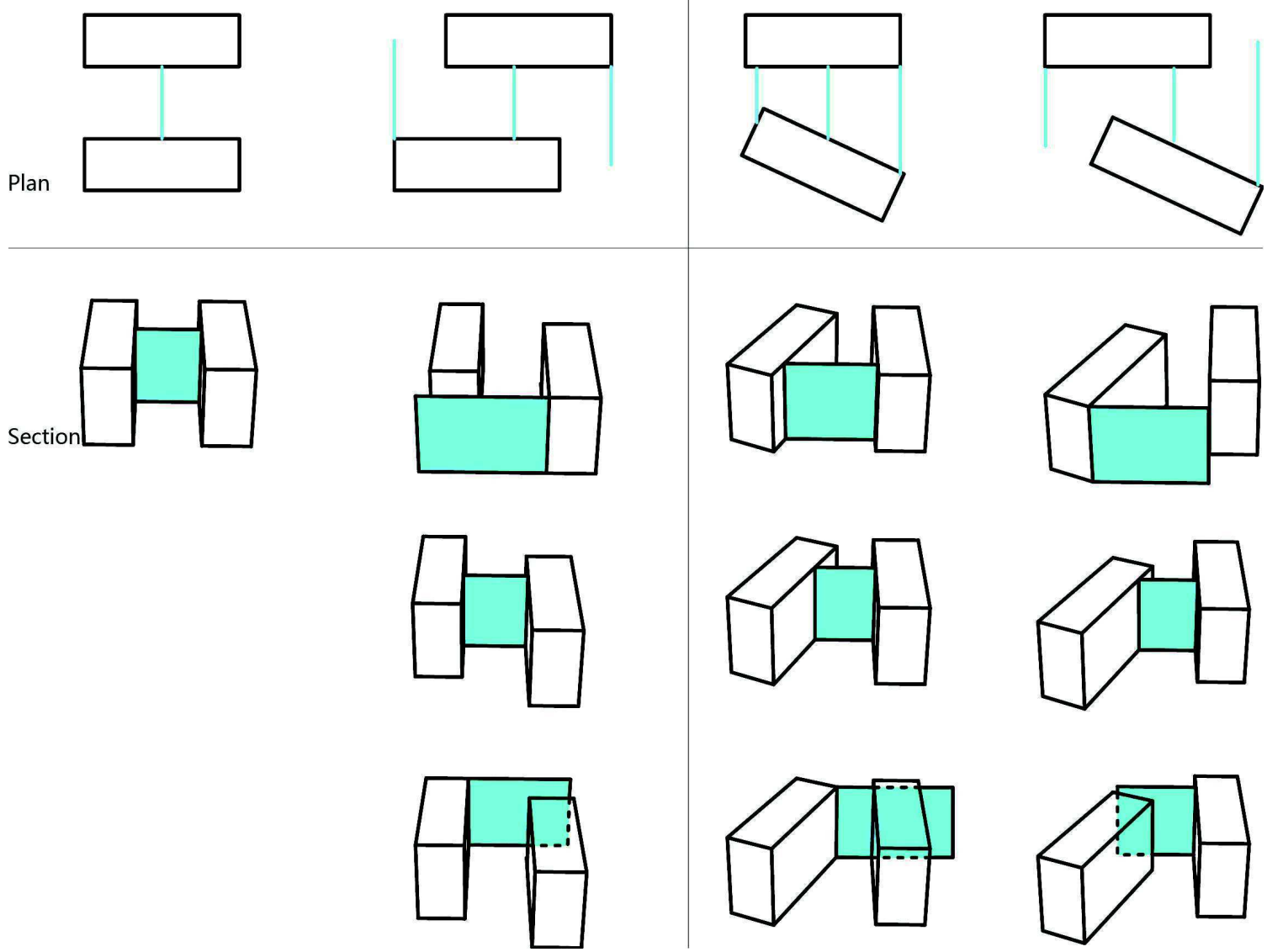

Figure 1.

Ideal patterns

producing secondary pollution. Therefore, this paper takes the windward velocity as the evaluation standard.

\section{Criteria for selecting residential Pattern}

The residential patterns have several forms: 'pavilion', 'street', 'court' and 'hybrid'.

Based on Western control indexes, China combines the actual situation and living habits of residents to introduce laws and regulations to limit the shape and pattern of apartment(including sunshine distance, fire separation, green rate and floor area rate). Among them, the floor area rate is to determine developers' economic interests. In order to maximize this index, patterns of China's residential areas are dominated by 'street' and 'point' types.

This paper select the most common type of residential buildings, slab, to study. Different combinations of residential units form different types of slabs, in order to simplify classification, this paper refer to Zhao Qin (2015) which defines patterns of apartments according to the research methods of urban morphology, for slabs, patterns are measured in a combination of apartments' orientations. Single apartment or many apartments parallel to each other as one orientation type, apartments with different orientations are multi-orientation type.

One orientation and multi-orientation also include two different sub-types. The first subtype is that two buildings on both sides are aligned. The second sub-type is that the two buildings are staggered, some parts are not blocked by adjacent buildings and result in a gap. Each type has different section types, and therefore various ratio of building height to building spacing $(\mathrm{H} / \mathrm{W})$, and as heights increase, the ratio varies with each type, therefore, there is a very wide variation in the ratio.( Figure 1.) 


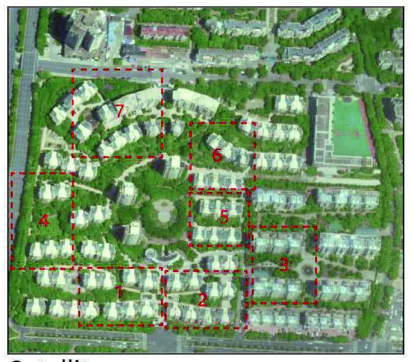

Satellite map

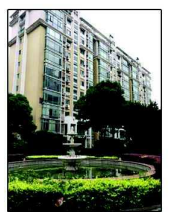

Picture

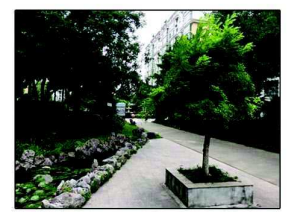

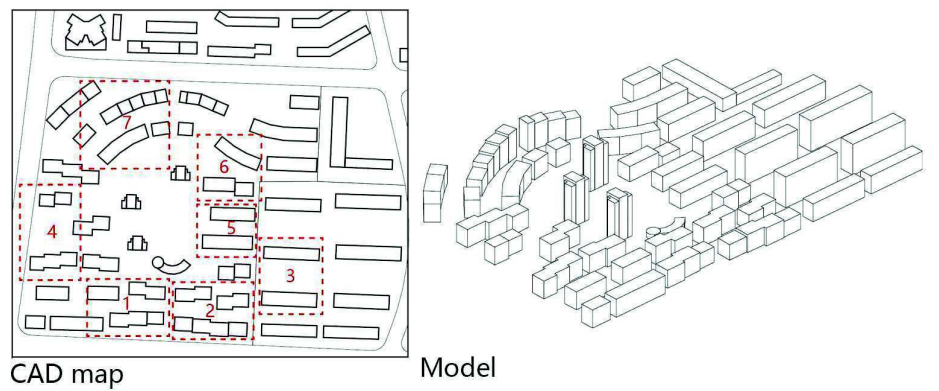
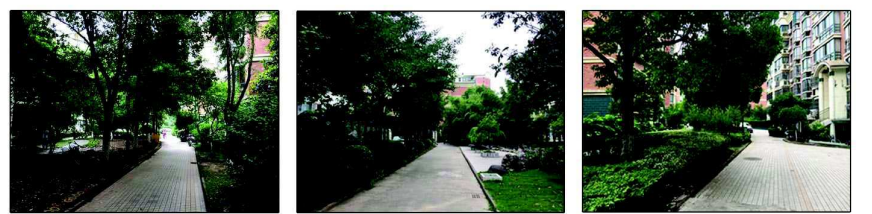

Figure 2.

Research area status

\section{Case study}

Case selection

The study area was selected in Hexi District of Nanjing City, near Yangtze River. It is a slice of $800 \mathrm{~m} * 1200 \mathrm{~m}$ restricted by Yangtze River Avenue, River northeast road, Cao Changmeng Street, Qing Liangmen Street. The slice is mainly residential, comprising a small number of public buildings and commercial buildings. This slice consists of 14 blocks, each block containing 1-4 different residential areas. Most buildings are multi-stories(from 6-9) buildings, and there are some high-rise buildings. Cad data comes from the Nanjing City Planning Bureau, some of which have been revised according to the latest Google earth satellite map.

The simulation of wind environment for the entire $800 \mathrm{~m} * 1200 \mathrm{~m}$ slice would take a lot of time and the sample capacity of wind speed data is difficult to meet requirements. So the range of simulation is reduced to the plot scale, and this paper would evaluate the first plot (figure $\mathrm{xx}$ ). This plot completely covers the 4 ideal types summarized in chapter 2 , and according to the ideal types, seven regions are selected for evaluation and data analysis.( Figure 2.)

\section{CFD model set up}

The lateral and inflow boundaries are set to be
$5 \mathrm{H}$ ( $\mathrm{H}$ is the height of the highest building) away from the buildings, and the outflow boundary, $10 \mathrm{H}$. The height of the computational domain is $6 \mathrm{H}$. Therefore, the blockage ratio is less than $3 \%$, as recommended by Franke et al. (2006) and Tominaga et al. (2008). Totally 1,854,670 tetrahedral grids are generated in this study. The grid sensitivity analysis shows the errors caused by grid resolutions have an unnoticeable effect on the numerical results.

In this investigation, the power law inlet profiles are set as the inflow boundary conditions (Tominaga et al., 2008)

The above settings guarantee the authenticity and accuracy of the simulation, and the simulated wind speed diagrams are as follows (Figure 3).

\section{Analysis}

GIS platform for display of enhanced wind environment

In traditional CFD platform, wind speed is scalar data, unable to judge the direction of wind. However, the simulation results can be exported as coordinates with direction. After attaining wind speed values in specific area, GIS platform is used to enhance the display of wind data. GIS platform has a natural advantage in data visualization. 
It is convenient to gather data in different directions together to display.

Besides, data in GIS can be associated with geographic location of model, thus, the location information of a healthy wind environment region can be accurately obtained. Dr. Tang Lian in our team did some visualization experiment with simulation data on GIS platform in the early stage.

Wind speed data was visualized in $\mathrm{x}, \mathrm{y}, \mathrm{z}$ directions and she got ideal results. Therefore, this paper use this visualization system as well

\section{GIS displayed set up}

The simulation data of wind speed is imported in GIS platform. Then, select the same coordinate system with simulation and display the value of each coordinates sampling points with actual coordinates information.

Based on the wind speed of downwind (in this study, the direction of downwind is the same with South wind), the values are divided into 10 levels, warm color presenting downwind velocity, cold color presenting upwind velocity (Figure 4).

The calculation and filtering of form elements by MATLAB platform

The advantage of the MATLAB platform lies in the transformation between data and graphics. If it contains enough information, data can be converted into 2-dimensional or 3-dimensional models, and it can process large amounts of data based on the user's own set of rules quickly. Our team wrote a processing program in 2013 in MATLAB platform.

It was based on coordinates and height information of each corner point of imported building. Then building information modeling was re-established in MATLAB and filtered by 0.7 times and 0.4 times of building height. The data of windward and leeward side can be calculated under different wind direction. On this basis, this paper further improve this program to calculate ratio of building height to building spacing in different positions and heights.

\section{MATLAB model set up}

Import the CAD data into GIS platform and extract corner coordinates, height information, and building codes for each building, then import the MATLAB platform. Select the number of each building with minimum $\mathrm{Y}$ coordinates. With this corner as the starting point, connect each corner to the counter counterclockwise. If the $\mathrm{X}$ coordinate of starting point of a line is greater than it at the end point, this line is back side edge, on the contrary, the line is front edge. Then, calculate ratio of building height to building spacing at each front and corresponding back side edge (Figure 5.).

\section{Data analysis}

According to the data calculated by GIS and MATLAB, the plot of W/H-downwind velocity is plotted. (Using $\mathrm{W} / \mathrm{H}$ instead of $\mathrm{H} / \mathrm{W}$, because $\mathrm{W} / \mathrm{H}$ increases with height and could get better effect of plot)( Figure 6-2.)

The downwind velocity corresponds to the $\mathrm{y}$ coordinate value at the right of each chart, the $\mathrm{W} / \mathrm{H}$ corresponds to the $\mathrm{y}$ coordinate value at the left of each chart. The $\mathrm{x}$ coordinate is the number of the area in which each data is located.

In general, with the increase of $\mathrm{W} / \mathrm{H}$, the downwind velocity is also increasing, the wind environment has improved significantly since the sixth storey, $\mathrm{W} / \mathrm{H}$ also rises to a relatively large value, and a similar ratio does not necessarily produce similar velocity. Then make statistics on all ratios and velocity. ( Figure 6-3.)

There are some statistical data. SSE:1107 (The closer the value is to 0 , the better the fitting effect;)R-square: 0.04974 (The closer the value is to 1 , the better the fitting effect). Therefore, there is no linear correlation between the overall $\mathrm{W} / \mathrm{H}$ ratio and the corresponding downwind velocity.

Next, make statistical analysis between $\mathrm{W} / \mathrm{H}$ ratio and the corresponding downwind velocity in the similar region, a similar region refers to the relative relation of adjacent buildings and layout of the surrounding buildings. (Figure 

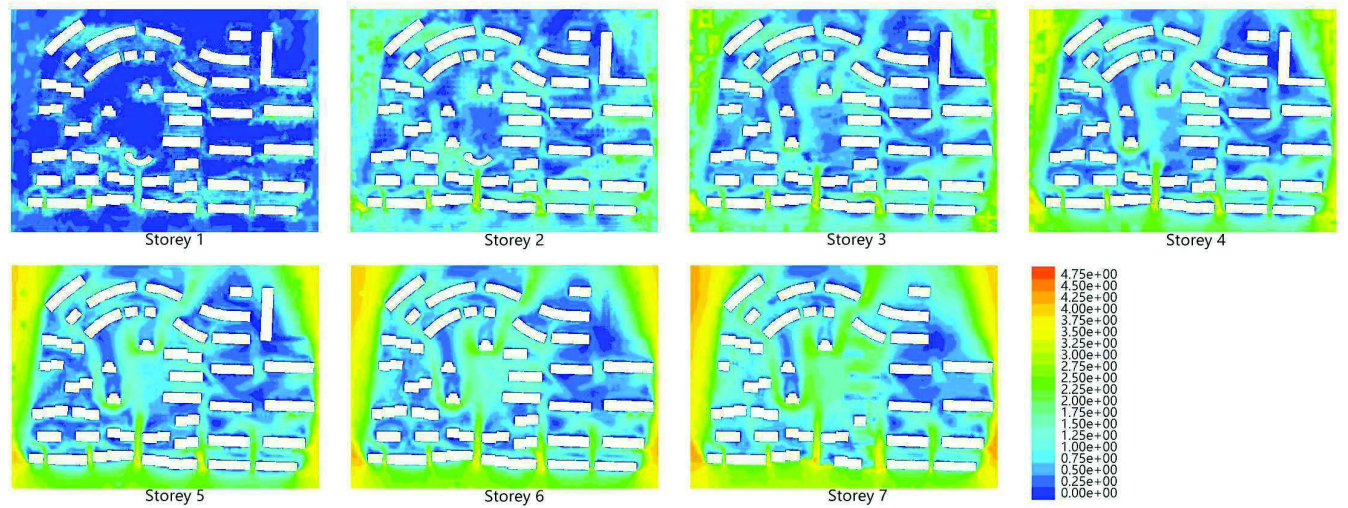

Figure 3.

Simulation results
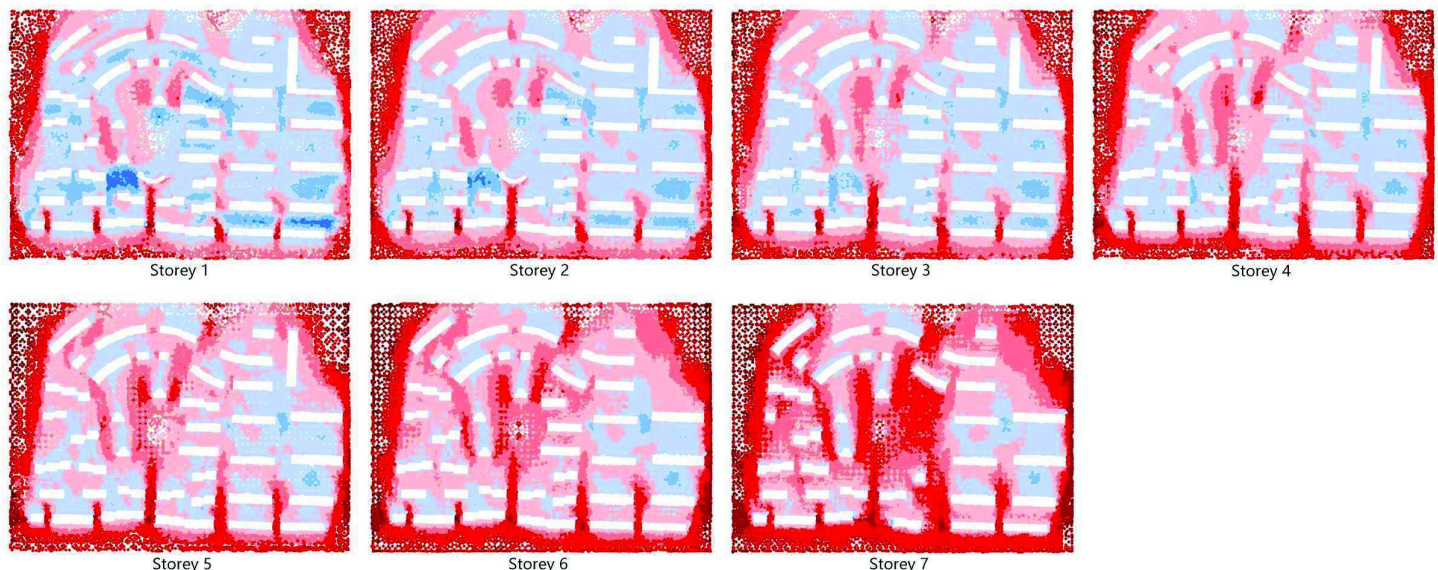

Figure 4.

GIS results
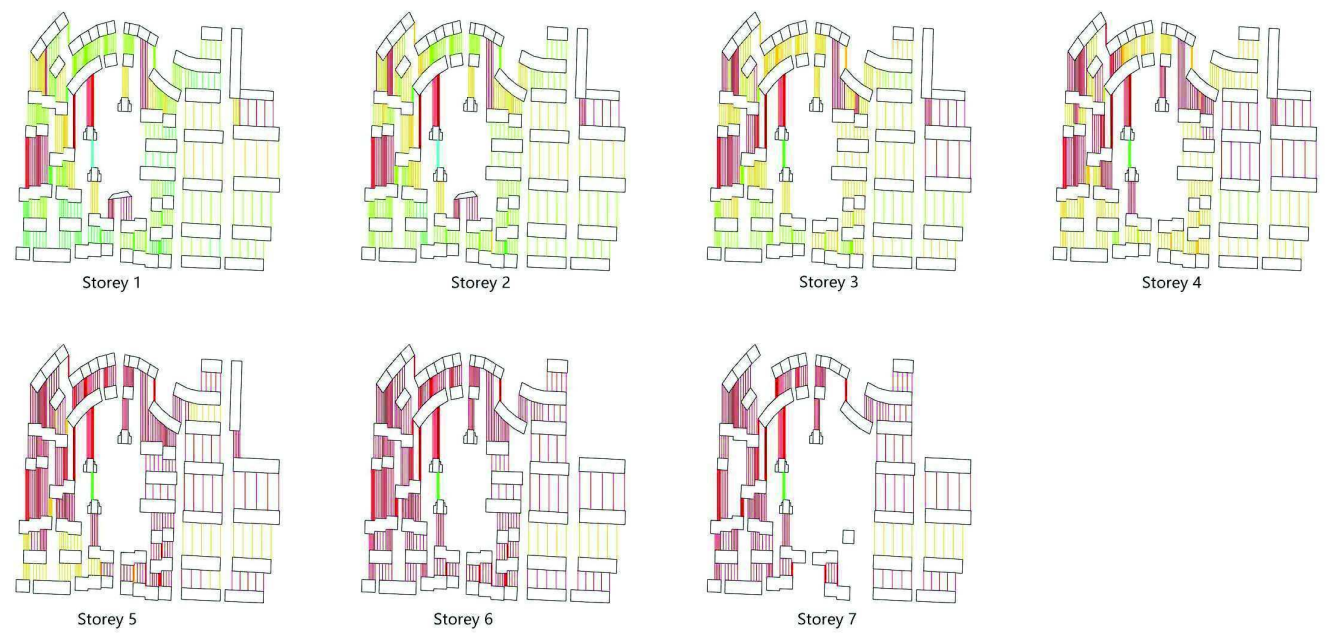

Figure 5.

MATLAB results 

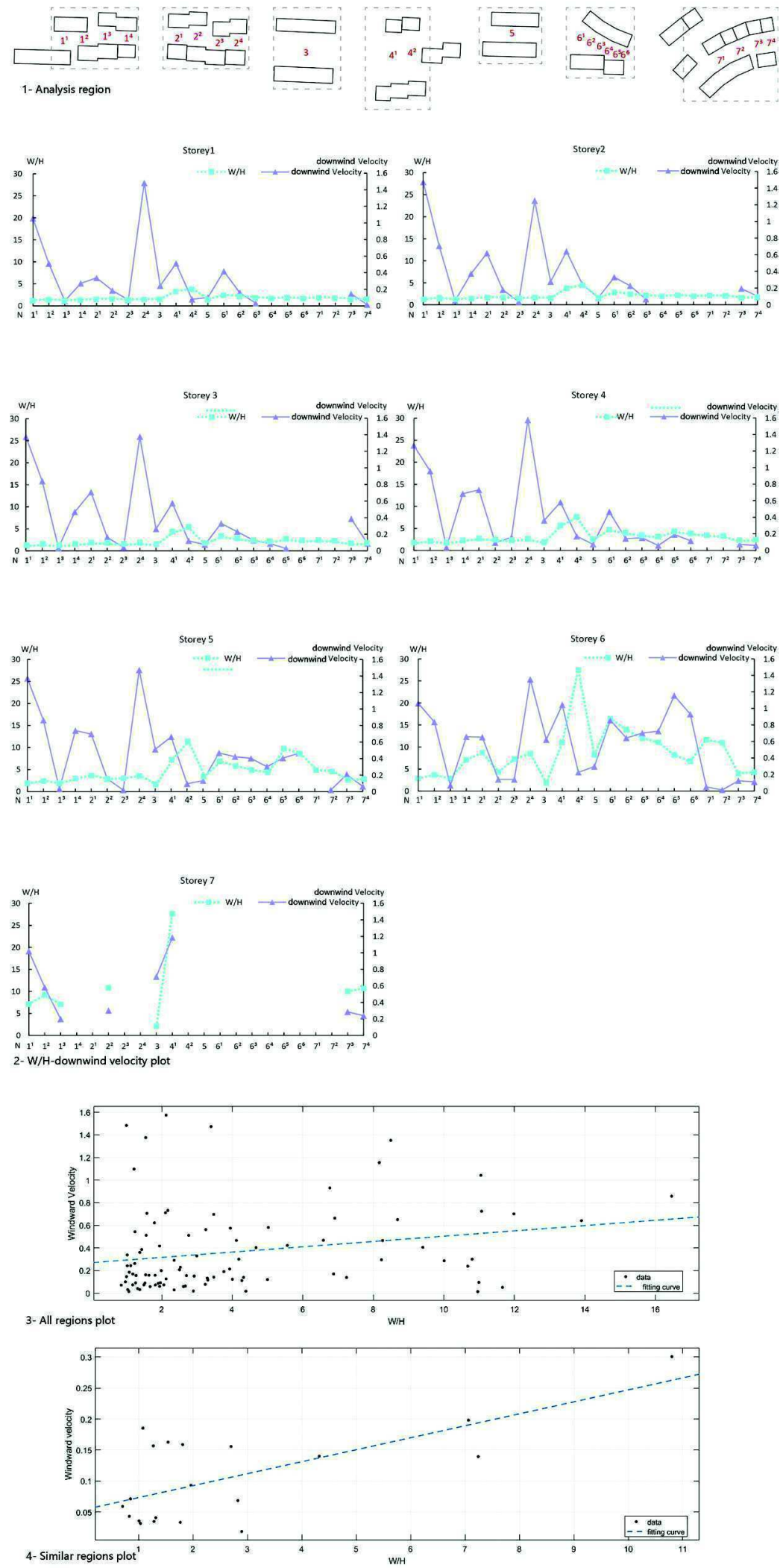

Figure 6.

Data analysis 
6-1. $\left.\left(1^{3}, 2^{2}, 2^{3}\right)\right)$ SSE: 0.05673; R-square: 0.473 From this we can see that there is a linear correlation with partial probabilities between the $\mathrm{W} / \mathrm{H}$ ratio and the downwind velocity in the similar area. ( Figure 6-4.)

Then, a longitudinal comparison of downwind velocity and $\mathrm{W} / \mathrm{H}$ ratio is made. Region1 (number $1^{1}-1^{4}$ ), buildings in this area are basically perpendicular to the simulated wind direction and are staggered one orientation pattern. Two distinct narrow corridors of wind have been formed. Under similar $\mathrm{W} / \mathrm{H}$ ratio, the velocity is always greater in the area near the corridor that it off the corridor.

Region2 (number $2^{1}-2^{4}$ ), buildings in this area are basically perpendicular to the simulated wind direction and are one orientation pattern with lateral spacing. And the results are similar to Region 1.

Region3 (number3), buildings in this area are basically perpendicular to the simulated wind direction and are one orientation pattern with aligned lateral sides. The $\mathrm{W} / \mathrm{H}$ ratio is always within 2.5 , and the velocity increases gradually with the rise of this ratio.

Region4 (number $4^{1}-4^{2}$ ), buildings in this area are basically perpendicular to the simulated wind direction and are one orientation pattern with extremely large longitudinal spacing. The $\mathrm{W} / \mathrm{H}$ ratio is always beyond 3 , with the rise of $\mathrm{W} / \mathrm{H}$ ratio, the velocity is also rising, but there is no obvious proportional relationship between the sets of data.

Region5 (number5), buildings in this area are basically perpendicular to the simulated wind direction and are one orientation pattern. Velocity increased slowly with the increase of $\mathrm{W} / \mathrm{H}$ ratio, and the velocity increased significantly at six storey when the ratio was greater than 8 .

Region6 (number6 ${ }^{1}-6^{6}$ ), buildings in this area has an angle about 60 degrees with the simulated wind direction(counterclockwise) and are staggered multi-orientation pattern. The longitudinal spacing between buildings changes obviously. At the same height, where the $\mathrm{W} / \mathrm{H}$ ratio is large, the velocity is greater.

Region7 (number $7^{1}-7^{4}$ ), buildings in this area has an angle about 60 degrees with the simulated wind direction(clockwise) and are one orientation pattern at this angle. The
$\mathrm{W} / \mathrm{H}$ ratio increased significantly but the velocity increased slowly. When the one shelter disappears, the velocity in this region is improved greatly.

On the whole, Region 1,2 and 4 always have better wind environment. Morphological features are as follows: The lateral spacing of region 1 and 2 is small, forming a number of wind corridors, greatly improving the wind environment in the regions. Region4 has large longitudinal spacing so the $\mathrm{W} / \mathrm{H}$ ratio is much greater than ordinary patterns of residential areas.

\section{Conclusion}

For patterns which meet the needs of Chinese laws and regulations, with the height increasing and the $\mathrm{W} / \mathrm{H}$ ratio increasing, the wind environment will gradually improve, however, the magnitude of the improvement in different regions is not the same.

For all $\mathrm{W} / \mathrm{H}$ ratio and downwind velocity in all different regions, these did not produce a simple linear correlation, the length of buildings, the formation of wind corridors, the angle of buildings with the simulated wind direction may all have an impact on the downwind velocity.

For $\mathrm{W} / \mathrm{H}$ ratio and downwind velocity in similar regions, it is assumed that there is a linear correlation between certain probabilities. In the next experiment, we need to add experimental samples and control experimental variables to obtain more accurate conclusions.

\section{References}

Buccolieri, R., Sandberg, M. and Sabatino, S. D. (2010) City breathability and its link to pollutant concentration distribution within urban-like geometries, Atmospheric Environment 44, 1894-1903

Matteo Carpentieri, Alan G. Robins.(2015) Influence of urban morphology on air flow over building arrays, Journal of Wind Engineering and Industrial Aerodynamics 145

Hu, T., \& Yoshie, R. (2013). Indices to evaluate 
ventilation efficiency in newly-built urban area at pedestrian level. Journal of Wind Engineering \& Industrial Aerodynamics, 112(1), 39-51.

Bady, M., Kato, S., \& Huang, H. (2008). Towards the application of indoor ventilation efficiency indices to evaluate the air quality of urban areas.Building \& Environment, 43(12), 1991-2004.

Yang, F., Qian, F., \& Lau, S. S. Y. (2013). Urban form and density as indicators for summertime outdoor ventilation potential: a case study on high-rise housing in shanghai. Building \& Environment, 70(12), 122-137.

Mfula, A. M, Kukadia, V., Griffiths, R. F. and Hall, D. J. (2005) Wind tunnel modelling of urban building exposure to outdoor pollution, Atmospheric Environment 39, 2737-2745

Oke T. R. (1988) Street design and urban canopy layer climate, Energy and Buildings 11, 103-111.

Chan, A. T., So E. S. P. and Samad S. C. (2001) Strategic guidelines for street canyon geometry to achieve sustainable street air quality, Atmospheric Environment 35, 4089-4098

Simoëns, S. and Wallace, J. M. (2008) The flow across a street canyon of variable widthPart 2: Scalar dispersion from a street level line source, Atmospheric Environment 42, 2489-2503

Buccolieri, R., Salizzoni, P., Soulhac, L., Garbero, V. and Sabatino, S. D. (2015) The breathability of compact cities, Urban Climate 13, 73-93

You Wei, Wowo Ding (2016) Assessment of outdoor space's ventilation efficiency around residential building: Effects of building dimension, separation and orientation,50th International Conference of the Architectural Science Association 2016, pp. 1-10.. 